\title{
A detailed study of the configuration selected multireference configuration interaction method combined with perturbation theory to correct the wave function
}

\author{
B. Engels \\ Institut für Physikalische und Theoretische Chemie, Universität Bonn, Wegelerstrasse 12, \\ 53115 Bonn, Germany
}

(Received 18 August 1993; accepted 22 September 1993)

\begin{abstract}
A reliable prediction of the isotropic hyperfine coupling constant $A_{\text {iso }}$ is still a difficult task for $a b$ initio calculations. In previous studies, the configuration selected multireference configuration interaction method in combination with perturbation theory to correct the wave function ( $M R C I / B_{K}$ ) yielded accurate isotropic hyperfine coupling constants very economically. The present study gives a detailed analysis of the $\mathrm{MRCI} / B_{K}$ method based on the $X^{2} \Pi$ state of $\mathrm{CH}$ as a test case. Furthermore, a comparison to various other methods such as Møller-Plesset perturbation theory and the coupled cluster approach is made. The success of the $\mathrm{MRCI} / B_{K}$ method in predicting isotropic hyperfine coupling constants is explained in terms of the influence of higher than double excitations.
\end{abstract}

\section{INTRODUCTION}

The magnetic interaction between the nuclear spin I and the electron spin $\mathbf{S}$ can be described by the hyperfine coupling constants (hfcc). It consists of an isotropic part and an anisotropic part. The isotropic part, also called the Fermi contact term, possesses no classical analog. It is proportional to the net unpaired electron-spin density at the nucleus and is given by the isotropic hyperfine coupling constant $A_{\text {iso }}$ defined as ${ }^{1}$

$$
A_{\text {iso }}(N)=\frac{8 \pi}{3} g \beta_{N} \beta_{g_{N}} \frac{1}{S}\left\langle\Psi\left|\sum_{k=1}^{n} \delta\left(r_{k}-r_{N}\right) s_{z}(k)\right| \Psi\right\rangle,
$$

where the term in the bra-ket is the total spin density $\left\langle\delta\left(r_{N}\right)\right\rangle$ of the electrons at the location of the nucleus $N$. The term $g$ is the $g$ value for the electrons in the radical, while $\beta_{N}$ is the Bohr magneton. In the present work, $g$ was set to the value for the free electron $g_{e}$. The quantities $g_{N}$ and $\beta_{N}$ are the nuclear $g$ factor and the value for the nuclear magneton, respectively.

The anisotropic part describes the dipole-dipole interaction between $I$ and $S$. Its Cartesian components are defined in a molecule-fixed coordinate system as ${ }^{1}$

$A_{i j}(N)=g_{N} g \beta_{N} \beta_{e} \frac{1}{S}\left\langle\Psi\left|\sum_{k=1}^{n}\left(\frac{3 i j-r^{2} \delta_{i j}}{r^{3}}\right)_{c k} 2 s_{z k}\right| \Psi\right\rangle$

with $i, j=x, y, z ; c k$ indicates that $A_{i j}$ is formulated with respect to the center $N$.

Since each nucleus with $I>0$ contributes to the splitting, the hyperfine spectrum is very complex, but opens the possibility of characterizing the molecule in different regions.

While the calculation of anisotropic hfecs is not too complicated, a reliable prediction of $A_{\text {iso }}$ is still a very difficult task for $a b$ initio calculations. The difficulties arise since only those orbitals which possess a nonvanishing value at the position of the nucleus in question and a net spin density contribute to $A_{\text {iso }}$. Thus spin polarization effects have to be taken into account. Very often all doubly occupied shells contribute to $A_{\text {iso }}$. Since the contributions from the shells are similar in magnitude, but differ in sign, a balanced description of the correlation effects for all shells is essential. This explains the strong dependence of $A_{\text {iso }}$ on the atomic orbital (AO) basis and on the quality of the configuration interaction (CI) wave function, as has been seen previously. ${ }^{2-6}$

With respect to the quality of the CI wave function, recent investigations ${ }^{3,4,7,8}$ emphasize the importance of higher than double excitations. A good example is the ground state $\left(X^{2} \Pi\right)$ of the $\mathrm{CH}$ molecule. ${ }^{8}$ For the isotropic hfcc of the carbon center $A_{\text {iso }}\left({ }^{13} \mathrm{C}\right)$, very good agreement with the experimental value of $46.8 \mathrm{MHz}( \pm 2.8$ $\mathrm{MHz}$ ) can only be obtained if triple excitations are taken into account (45 $\mathrm{MHz})$. A single and double excitation configuration interaction (SDCI) gives a value of $30 \mathrm{MHz}$, i.e., an error of more than $30 \%$. Surprisingly a CI which includes only single excitations with respect to the Hartree-Fock configuration (SCI) gives much better agreement ( $41 \mathrm{MHz}$ ) than SDCI calculations. The SCI is a subspace of the SDCI, so that some sort of error cancellation has to be expected.

A more founded understanding of the influences of the various excitation classes is given in our recent study ${ }^{9}$ in which the effects are divided into an indirect and a direct part. Going from a SCI to a SDCI, the double excitations can influence $A_{\text {iso }}$ in two ways. ${ }^{5}$ First, a direct effect arises from the coefficients of the double excitations themselves, which are not in the $\mathrm{SCI}$ wave function. A second influence of the double excitations on $A_{\text {iso }}$ is of more indirect nature. Due to interactions within the SDCI Hamilton matrix among configurations already included in the $\mathrm{SCI}$ and the double excitations, the coefficients of the restricted Hartree-Fock (RHF) configuration and of the single excitations obtained by a SDCI treatment differ from those obtained by the SCI treatment. From these modifications 
to the coefficients, a further change in $A_{\text {iso }}$ results. It can also be traced back to the influence of the double excitations, and in the following it will be called the indirect effect of the double excitations on $\boldsymbol{A}_{\text {iso }}$. The indirect effect contains both normalization effects and changes in the ratio among the individual coefficients, but the latter are found to be much more important.

The study shows clearly that, at least for the studied systems (boron, carbon, and nitrogen atoms), the effects of the higher excitations (triples and quadruples) arise mainly due to indirect effects, while direct effects of the higher excitations are quite small. Therefore, a procedure which would be able to include the indirect effects of less important configurations should be able to give very reliable hfecs.

In a previous paper, we used the $B_{K}$ method $^{10}$ in a modified form $^{5}$ in combination with a configuration selected multireference configuration interaction (MRCI) method to calculate the isotropic hfecs of nitrogen and boron atoms. This approach was found to be able to cover the indirect effects of less important configurations not handled variationally. In the present paper, hfcc calculations for a system which is well-known for the difficulties in the calculations of isotropic $\mathrm{hfccs}^{6,11}$ are presented. After a short review of the technical details, a study of the ground states of $\mathrm{CH}\left(X^{2} \Pi\right)$ will be discussed. A comparison with various methods taken either from the literature or calculated using the Gaussian88 program $^{12}$ will be given. One important question in connection with the $B_{K} / A_{K}$ approach is how the isotropic hfecs or any other property calculated with the perturbationally corrected wave function depends on the number of configurations already treated variationally, i.e., included explicitly in $H_{0}$. The calculations of the correction terms being quite expensive, the question of how many configurations actually have to be included within the $B_{K} / A_{K}$ step also arises. Both questions will be discussed in the present study.

\section{THEORY}

The theory of the $B_{K}$ method is based on partitioning perturbation theory. ${ }^{13,14}$ Suppose the Hamilton matrix $\mathbf{H}$ of the MRCI space is partitioned as

$$
\left(\begin{array}{cc}
\mathbf{H}_{0} & \mathbf{h}^{\mathbf{T}} \\
\mathbf{h} & \mathbf{H}_{1}
\end{array}\right)\left(\begin{array}{l}
c_{0} \\
c_{1}
\end{array}\right)=E\left(\begin{array}{l}
c_{0} \\
c_{1}
\end{array}\right)
$$

where $\mathrm{H}_{0}$ is a $N \times N$ submatrix of $\mathrm{H}$ containing all important configurations. Then $\mathbf{H}_{1}$ is a $(K-N) \times(K-N)$ matrix formed from configurations of lesser importance and $h$ contains the connecting matrix elements between the two sets of configurations. Within selective MRCI techniques, $h$ and $H_{1}$ are neglected and only

$$
\mathrm{H}_{0} \mathrm{c}_{0}^{\prime \prime}=E \mathrm{c}_{0}^{\prime \prime}
$$

is solved. The formula of the $B_{K}$ method according to Davidson $^{15-18}$ is obtained if Eq. (3) is written as

$$
\left[\mathrm{H}_{0}+\mathrm{h}^{\mathrm{T}}\left(E-\mathrm{H}_{1}\right)^{-1} \mathrm{~h}\right] \mathrm{c}_{0}=E \mathrm{c}_{0}
$$

and $H_{1}$ is replaced by its diagonal part

$$
\left[\mathbf{H}_{0}+\mathbf{h}^{\mathrm{T}}\left(E^{\prime}-\mathrm{D}\right)^{-1} \mathbf{h}\right] \mathbf{c}_{0}^{\prime}=E \mathbf{c}_{0}^{\prime} \text {. }
$$

Depending on how $E^{\prime}$ in Eq. (6) is chosen, one arrives at Brillouin-Wigner ( $E^{\prime}$ is equal to $E$ and the equation is solved iteratively) or Rayleigh-Schrödinger [ $E^{\prime}$ is equal to the energy in Eq. (4)] perturbation theory. ${ }^{15,16}$ In the present work, Rayleigh-Schrödinger perturbation theory was adopted. The indirect effect of the neglected configuration is accounted for because the new vector $\mathbf{c}_{0}^{\prime}$ contains the relaxation of $c_{0}^{\prime \prime}$ due to the neglected configurations in second-order perturbation theory.

The coefficients of configurations not contained in $c_{0}^{\prime}$ can also be estimated in second-order perturbation theory using the $A_{K}$ method

$$
\mathbf{c}_{\mathbf{1}}^{\prime}=(\mathbf{1} E-\mathrm{D})^{-1} \mathbf{h c}_{\mathbf{0}}^{\prime} \text {. }
$$

In the present study, the efficiency of the $B_{K}$ method in correcting a truncated $\mathrm{CI}$ wave function is tested. A calculation consists of two steps. After choosing the reference space, all single and double excitations are generated. In the first step, the $\mathbf{H}_{\mathbf{0}}$ matrix is diagonalized. It contains all configurations which lower the energy by more than a given threshold $T_{\mathrm{CI}}$. In the second step, the $B_{K}$ method is applied. Since the computation of all terms in $\mathbf{h}^{\mathbf{T}}\left(E^{\prime}-D\right)^{-1} \mathbf{h}$ is very time consuming, they are only calculated for the most important configurations. All configurations possessing a coefficient absolutely greater than a given threshold $T_{B K}$ were included in the $B_{K}$ correction.

\section{RESULTS AND DISCUSSION}

The calculation of the isotropic hfcc of the carbon center $A_{\text {iso }}\left({ }^{13} \mathrm{C}\right)$ in the $X^{2} \Pi$ of $\mathrm{CH}$ is a great challenge for $a b$ initio calculations, although only seven electrons need to be correlated. The $X^{2} \Pi$ state is derived from the electronic configuration $1 \sigma^{2} 2 \sigma^{2} 3 \sigma^{2} 1 \pi^{1}$. Since only the $\pi$ orbital is singly occupied, the restricted Hartree-Fock (RHF) approach yields a value of zero for the isotropic hfecs of both centers. The correct value [the experiment ${ }^{19,20}$ gives (46.8 $\pm 2.8 \mathrm{MHz})$ for the carbon center and $(-57.7 \pm 0.3$ MHz) for the hydrogen center] is determined by correlation and/or polarization effects which can be taken into account for example by a MRCI treatment.

For the study of the $\mathrm{CH}$ molecule, the two different AO basis sets given in Table I were used. The smaller AO basis set $(13 s 8 p 3 d 1 f / 9 s 3 p) \rightarrow[8 s 5 p 3 d 1 f / 6 s 3 p]$ was used to perform less expensive calculations. Experience gained from this basis set was used in calculations with the larger basis set $(14 s 9 p 5 d 1 f / 10 s 3 p) \rightarrow[9 s 6 p 5 d 1 f / 7 s 3 p]$. The latter is flexible enough for a very reliable calculation of isotropic hfces. All calculations were performed for $R_{e}=1.118 \AA$.

Let us first focus on the results obtained with the smaller basis set. To test the quality of the $B_{K} / A_{K}$ approximation, we compared the isotropic hfces obtained with the $B_{K} / A_{K}$ scheme with the exact limit, given by $A_{\text {iso }}$ calculated with the unselected MRCI wave function. This was possible for a smaller reference space (six configurations) leading to a total MRCI space of 171901 configurations. The MRCI calculations were performed with the MELDF-X program package. ${ }^{22}$ The calculated values of 
TABLE I. The description of $\mathrm{AO}$ basis sets used for the $\mathrm{CH}$ molecule.

\begin{tabular}{|c|c|c|}
\hline \multicolumn{3}{|c|}{ Small AO basis } \\
\hline Carbon center & $\begin{array}{l}(13 s 8 p) \rightarrow[8 s 5 p] \\
+3 d \text { function }(2.292 / 0.838 / 0.292) \\
+1 f \text { function }(0.761)\end{array}$ & Duijneveldt (Ref. 21) \\
\hline Hydrogen center & $\begin{array}{l}(8 s) \rightarrow[5 s] \\
+1 s \text { function }(2.4) \\
+3 p \text { function }(1.848 / 0.649 / 0.228)\end{array}$ & Duijneveldt (Ref. 21) \\
\hline \multicolumn{3}{|c|}{ Large AO basis } \\
\hline Carbon center & $\begin{array}{l}\text { Small basis } \\
+1 s(0.02) \\
+1 p(0.0358) \\
+2 d(8.0 / 0.1)\end{array}$ & Ref. 31 \\
\hline Hydrogen center & $\begin{array}{l}\text { Small basis } \\
+1 s(0.01)\end{array}$ & Ref. 31 \\
\hline
\end{tabular}

$A_{\text {iso }}\left({ }^{13} \mathrm{C}\right)$ as a function of the number of configurations handled variationally are given in Fig. 1. Because of their importance for one-electron properties, all single excitations were not subject to the selection procedure, i.e., all single excitations are always included in $\mathbf{H}_{\mathbf{0}}$. For these test calculations, self-consistent field molecular orbitals (SCFMOs) were used as the one-particle basis. If the truncated MRCI wave function without corrections is used, a strong dependence of $A_{\text {iso }}\left({ }^{13} \mathrm{C}\right)$ on the number of configurations handled variationally is found. First, if the number of configurations is smaller than about 10000 , a decrease in $A_{\text {iso }}\left({ }^{13} \mathrm{C}\right)$ is found. For $H_{0}$ ranging in size from 10000 to 30000 configurations, the value of $A_{\text {iso }}\left({ }^{13} \mathrm{C}\right)$ increases strongly, while for larger $H_{0}$, slow convergence to the exact MRCI value is seen. Similar behavior for the isotropic hfccs of the heavier center was also found for $A_{\text {iso }}\left({ }^{14} \mathrm{~N}\right)$ in the ground state $X^{3} \Sigma^{-}$of $\mathrm{NH}^{23}$ To push the error below $5 \%(1.2 \mathrm{MHz})$, it is necessary to include $20 \%-30 \%$ of the total MRCI space. The value of $A_{\text {iso }}\left({ }^{13} \mathrm{C}\right)$ obtained with

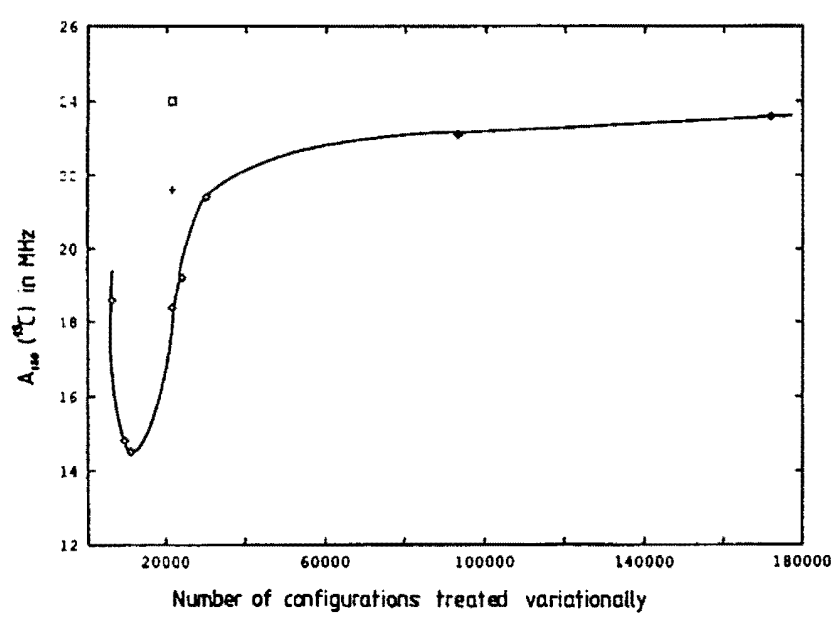

FIG. 1. A comparison of the $B_{K} / A_{K}$ method with MRCI calculation. The points denote $A_{i \infty}^{\mathrm{C}}\left({ }^{13} \mathrm{C}\right)$ obtained from truncated MRCI calculations. $B_{K} / A_{K}$ were performed for $T_{\mathrm{CI}}=10^{-7}$ (21 290 configurations). The isotropic hfec obtained with the $B_{K}$ method is indicated with $+;$ the values including the $B_{K}$ and the $A_{R}$ corrections are denoted by $\square$. the exact MRCI wave function is $23.6 \mathrm{MHz}$. A $B_{K} / A_{K}$ treatment was performed for $T_{\mathrm{CI}}=10^{-7}$ hartrees. With this threshold, 21290 configurations, i.e., $12.4 \%$ of the total MRCI space, were handled variationally. The indirect effect, estimated by the $B_{K}$ treatment, is $3.2 \mathrm{MHz}$, while the direct effect calculated with the $A_{K}$ method gives $2.4 \mathrm{MHz}$. Using perturbation theory, $A_{\text {iso }}\left({ }^{13} \mathrm{C}\right)$ is corrected from 18.4 to $24.0 \mathrm{MHz}$. This is about $0.4 \mathrm{MHz}(1.2 \%)$ higher than the exact MRCI value. To obtain similar agreement using the configuration selected MRCI method without any correction, it is necessary to include about $50 \%$ of the MRCI space ( 95000 configurations). The remaining differences to the experimental result of $46.8 \mathrm{MHz}$ are due to the neglect of higher excitations. The exact MRCI energy ( $T_{\mathrm{CI}}=0.0$ hartree) is -38.4510 hartrees. For $T_{\mathrm{Cl}}=10^{-7}$ hartree, a total energy of $-38.4497 \mathrm{har}$ trees is calculated. Using the energy extrapolation scheme developed by Peyerimhoff and Buenker, ${ }^{24}-38.4508$ hartrees are obtained, while the $B_{K}$ method gives -38.4512 hartrees. It is seen that both methods lead to very similar estimates of the exact MRCI energy, the former procedure being less expensive. However, it should be kept in mind that in this extrapolation scheme, only the energy is corrected, while the wave function remains unchanged.

For calculations using larger reference spaces which are very important for a reliable description of isotropic hfccs, our software was unable to handle the total MRCI space. Therefore, results will be compared with experimental data. To obtain faster convergence of the $\mathrm{CI}$ expansion, natural orbitals (NOs) were used as the one-particle basis. Besides the reliability of the method, the question of economy is also important. In connection with the $B_{K} / A_{K}$ approach, it is therefore interesting to investigate how the isotropic hfecs (or any other property) calculated with the perturbationally corrected wave function depend on the number of configurations already included in $\mathbf{H}_{0}$. To gain insight into the problem, we varied the size of $\mathrm{H}_{0}$. The influence of higher excitations was studied by increasing the size of the reference space. The reference configurations were selected according to the size of their coefficients. The results of the various calculations performed with the 
TABLE II. Details of calculations performed for the $X^{2} \Pi$ state of $C H$ using the small basis set. Energy is with respect to -34.0 hartrees, isotropic hfecs are in megahertz.

\begin{tabular}{|c|c|c|c|c|c|c|c|c|}
\hline$T_{\mathrm{Cl}^{b}}$ & SAF' & $E_{\mathrm{Cl}^{\mathrm{d}}}$ & $A_{\mathrm{iso}}^{\mathrm{Cl}}\left({ }^{13} \mathrm{C}\right)^{e}$ & $A_{\text {ino }}^{\mathrm{Cl}}(\mathrm{H})^{\mathrm{e}}$ & $E_{\text {MRD-CI }}{ }^{f}$ & $E_{B_{k}}$ & $A_{1 \times 0}^{8 X}\left({ }^{13} \mathrm{C}\right)^{8}$ & $A_{i=0}^{B_{K}}(\mathrm{H})^{z}$ \\
\hline \multicolumn{9}{|c|}{42 reference configurations $c^{2} \approx 0.980^{2} \mathrm{MR}$ space- $443464 \mathrm{SAF}$} \\
\hline 10.0 & 2366 & -0.4360 & 16.7 & -54.0 & -0.4544 & -0.4580 & 32.1 & -57.3 \\
\hline 1.0 & 8258 & -0.4486 & 31.1 & -55.3 & -0.4529 & -0.4559 & 41.8 & -57.7 \\
\hline 0.1 & 21947 & -0.4524 & 35.6 & -56.4 & -0.4541 & -0.4547 & 40.7 & -57.9 \\
\hline 0.05 & 29240 & -0.4529 & 35.0 & -56.8 & -0.4543 & & & \\
\hline \multicolumn{9}{|c|}{53 reference configurations $c^{2} \approx 0.984^{2} \mathrm{MR}$ space- $523413 \mathrm{SAF}$} \\
\hline 10.0 & 2894 & -0.4362 & 15.2 & -54.1 & -0.4541 & & & \\
\hline 1.0 & 8575 & -0.4487 & 31.2 & -55.0 & -0.4532 & -0.4562 & 41.8 & -57.3 \\
\hline 0.1 & 22582 & -0.4526 & 36.5 & -56.2 & -0.4545 & -0.4550 & 41.4 & -57.6 \\
\hline \multicolumn{9}{|c|}{72 reference configurations $c^{2} \approx 0.987^{\star} \mathrm{MR}$ space- $638893 \mathrm{SAF}$} \\
\hline 1.0 & 11255 & -0.4492 & 30.2 & -55.5 & -0.4538 & -0.4563 & 41.5 & -58.2 \\
\hline 0.1 & 24743 & -0.4527 & 35.3 & -56.4 & -0.4545 & -0.4553 & 41.6 & -58.2 \\
\hline
\end{tabular}

-Part of the reference space of the CI wave function on basis on the sum of the squares of coefficents.

b'In $10^{-6}$ hartrees.

The number of SAFs included in the diagonalization procedure.

dEnergy obtained by diagonalizing $\mathbf{H}_{0}$.

Tsotropic hfec calculated with the truncated MRCI wave function.

fCorrected using the MRDCI scheme.

Corrected using the $B_{K}$ scheme.

smaller AO basis set are given in Table II. In Fig. 2, the isotropic hfec of the carbon $A_{\text {iso }}\left({ }^{13} \mathrm{C}\right)$, which is the more sensitive property, is also plotted as a function of the various parameters.

Let us first consider $A_{\text {iso }}\left({ }^{13} \mathrm{C}\right)$. Using the uncorrected truncated MRCI wave function, the calculated value of $A_{\text {iso }}^{\mathrm{CI}}\left({ }^{13} \mathrm{C}\right)$ increases by about $15 \mathrm{MHz}$ if $T_{\mathrm{CI}}$ is lowered from $10^{-5}$ hartrees ( $\approx 3000$ configurations) to $10^{-6}$ hartrees ( $\approx 8000-9000$ configurations). If $H_{0}$ is further enlarged, $A_{\text {iso }}^{\mathrm{CI}}\left({ }^{13} \mathrm{C}\right)$ increases less rapidly, but it can be seen that convergence is not yet achieved at $T_{\mathrm{CI}}=5 \times 10^{-8}$

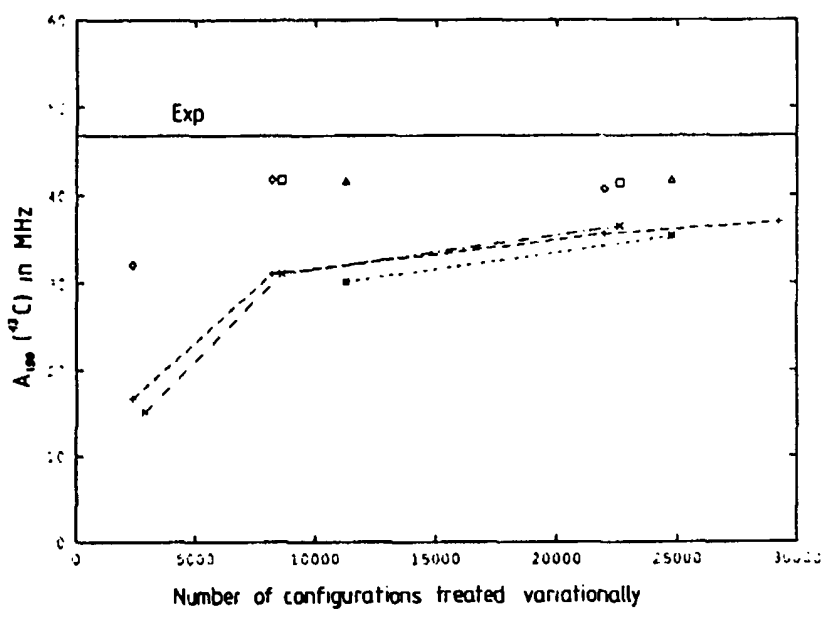

FIG. 2. Isotropic hfecs of the ${ }^{13} \mathrm{C}$ center calculated without and with $B_{K}$ corrections in dependence of the number of configurations handled variationally in the truncated $\mathrm{MRCI}$ calculation and the size of the reference space. All calculations were performed with the smaller basis set. The single calculations are indicated as follows: 42 reference configurations- - +--truncated MRCI, $\diamond$ including $B_{K} ; 53$ reference configurations-- $X--$-truncated MRCI, $\square$ including $B_{K} ; 72$ reference configuration- - $*---$ truncated MRCI, $\Delta$ including $B_{K}$.
(29 240 configurations). This behavior is quite similar to the dependencies found in other molecules ${ }^{6,23}$ and in the MO calculations discussed above. The lack of the decrease of $A_{\text {iso }}^{\mathrm{Cl}}\left({ }^{13} \mathrm{C}\right)$ for smaller sizes of $H_{0}$ as found for the MO calculations is due to the nature of the NOs. ${ }^{23}$ The dependence of $A_{\text {iso }}^{\mathrm{Cl}}\left({ }^{13} \mathrm{C}\right)$ on the number of configurations handled variationally is comparable for all reference spaces used in the present work. The decrease in $A_{\mathrm{iso}}^{\mathrm{CI}}\left({ }^{13} \mathrm{C}\right)$ going from 53 reference configurations to 72 reference configurations seems to be due to the selection criterion which tests the energy contribution of the single configurations, but not their importance for a given property. We will come back to this point later on.

Using the $B_{K}$ method, the indirect effect of the neglected configurations is taken into account. As expected, the $B_{K}$ method gives the largest correction for the smallest CI calculation $\left(T_{\mathrm{CI}}=10^{-5}\right.$ hartree; 2366 configurations handled variationally), where a correction of about 16 MHz is obtained for the isotropic hfec of the carbon center $\left[A_{\text {iso }}^{\mathrm{CI}}\left({ }^{13} \mathrm{C}\right)=16.7 \mathrm{MHz} ; A_{\text {iso }}^{B_{K}}\left({ }^{13} \mathrm{C}\right)=32.1 \mathrm{MHz}\right]$, but comparison with the calculation with smaller $T_{\mathrm{CI}}$ thresholds shows that the variationally handled space is too small. If more configurations are included in $H_{0}, A_{\text {iso }}^{B_{K}}\left({ }^{13} \mathrm{C}\right)$ shows little dependence on $T_{\mathrm{CI}}$. For the thresholds $T_{\mathrm{CI}}=10^{-6}$ and $10^{-7}$ hartrees, the value calculated with the corrected wave function $A_{\text {iso }}^{B_{K}}\left({ }^{13} \mathrm{C}\right)$ varies by about $1 . \mathrm{MHz}$, while $A_{\text {iso }}^{\mathrm{CI}}\left({ }^{13} \mathrm{C}\right)$ obtained from the normal truncated MRCI wave function changes by about $6 \mathrm{MHz}$. Furthermore, the corrected values are higher by about $5-10 \mathrm{MHz}$. The direct effect of the neglected configurations on $A_{\text {iso }}\left({ }^{13} \mathrm{C}\right)$ was studied using the $A_{K}$ method. The influence is less than 0.2 MHz independent of the size of $H_{0}$. This is in agreement with calculations at the boron atom which also show a small direct contribution if NOs rather than MOs are used. $^{5,9}$ 
TABLE III. Details of calculations performed for the $X^{2} \mathrm{II}$ state of $\mathrm{CH}$ using the large basis set. Energy is with respect to -34.0 hartrees, isotropic hfecs are in megahertz.

\begin{tabular}{|c|c|c|c|c|c|c|c|c|}
\hline$\overline{T_{\mathrm{Cl}}{ }^{b}}$ & SAF & $E_{\mathrm{Cl}^{d}}$ & $A_{i=0}^{C l}\left({ }^{13} C\right)^{e}$ & $A_{i=0}^{\mathrm{C}}(\mathrm{H})^{e}$ & $E_{\mathrm{MRD}} \mathrm{cl}^{\prime}$ & $E_{B_{k}}$ & $A_{i=0}^{B_{K}}\left({ }^{13} \mathrm{C}\right)^{6}$ & $A_{i s o}^{B_{K}}(\mathrm{H})^{2}$ \\
\hline \multicolumn{9}{|c|}{53 reference configurations $c^{2} \approx 0.984^{2} \mathrm{MR}$ space $-865680 \mathrm{SAF}^{\mathrm{h}}$} \\
\hline 1.0 & 13209 & -0.4506 & 33.2 & -55.1 & -0.4561 & -0.4595 & 44.4 & -57.6 \\
\hline 0.2 & 24066 & -0.4541 & 37.0 & -56.0 & -0.4566 & -0.4580 & 44.2 & -57.9 \\
\hline \multicolumn{9}{|c|}{77 reference configurations $c^{2} \approx 0.987^{*}$ MR space-1 $154750 S A F^{h}$} \\
\hline 1.0 & 13218 & -0.4508 & 33.8 & -55.1 & -0.4563 & -0.4597 & 46.0 & -58.2 \\
\hline 0.2 & 24135 & -0.4542 & 37.2 & -56.3 & -0.4567 & -0.4583 & 45.8 & -58.3 \\
\hline
\end{tabular}

"Part of the reference space of the CI wave function on basis on the sum of the squares of coefficents. In $10^{-6}$ hartrees.

The number of SAFs included in the diagonalization procedure.

'Energy obtained by diagonalizing $\mathbf{H}_{\mathbf{0}}$.

Tsotropic hfces calculated with the truncated MRCI wave function.

Corrected using the MRDCI scheme.

Corrected using the $B_{K}$ sheme.

${ }^{t}$ The number of corrected SAFs is $1500-2000\left(T_{B_{K}}=0.001\right)$.

The present study shows clearly that, for a reliable description of the isotropic hfccs, less than $2 \%$ of the total MRCI space has to be handled variationally if the effect of neglected configurations are taken into account using perturbation theory. The theoretically most sound value of $A_{\text {iso }}\left({ }^{13} \mathrm{C}\right.$ ) (72 reference configurations, 25000 configurations handled variationally, and 2000 configurations corrected perturbationally) is $41.4 \mathrm{MHz}$. The error with respect to experiment is about $11 \%$, i.e., the error of $20 \%$ within the truncated MRCI wave function was reduced by a factor of 2 .

The values calculated for the hydrogen center are given in Table II. The dependence on the quality of the calculation is smaller than was found for $A_{\text {iso }}\left({ }^{13} \mathrm{C}\right)$, but similar trends can be seen. The theoretically most sound value is $A_{\text {iso }}^{B_{K}}(\mathrm{H})=-58.3 \mathrm{MHz}$.

Before discussing the reasons for the success of the $B_{K}$ method, we will give a short description of our results obtained with the larger $\mathrm{AO}$ basis set. Several studies ${ }^{2,3,25,26}$ show that to obtain very accurate isotropic hfecs for atomic centers belonging to the second row, the inclusion of two compact $d$ functions in the basis set is necessary. This condition is fulfilled by the second, larger basis set. The results obtained with the larger basis set are given in Table III. The smaller reference space ( 52 configurations) corresponds to that used for the smaller basis set. The larger reference space ( 77 configurations) was optimized with respect to the spin density. For $A_{\text {iso }}\left({ }^{13} \mathrm{C}\right.$ ), the values calculated with the truncated MRCI wave function differ by about $1-2 \mathrm{MHz}$ from those obtained with the smaller AO basis set. The dependence of $A_{\text {iso }}\left({ }^{13} \mathrm{C}\right)$ on the size of the variationally handled space $H_{0}$ is comparable to that found for the smaller basis set, i.e., it increases by about 4 $\mathrm{MHz}$ if the number of variationally handled configurations is increased from about 13000 to about 24000 . The deviation from the experimental value is about $9 \mathrm{MHz}(20 \%)$. The difference between both reference spaces is quite small.

If the $B_{K}$ method is used, $A_{\text {iso }}^{B_{K}}\left({ }^{13} \mathrm{C}\right)$ depends only little on the underlying truncated MRCI calculation $(<0.3$ $\mathrm{MHz}$ ). The correction of $A_{\text {iso }}\left({ }^{13} \mathrm{C}\right.$ ) due to the $B_{K}$ method is about $8-9 \mathrm{MHz}$. The agreement of the theoretically most sound value ( 72 reference configurations, 24000 configurations handled variationally, and 1500 configurations corrected perturbationally) with the experimental value is excellent ( 45.8 vs $46.6 \mathrm{MHz}$ ), i.e., with the correction, the theoretical value lies within the experimental uncertainty ( $\pm 2.8 \mathrm{MHz}$ ).

As already seen for the smaller basis set, similar but less prominent trends are found for $A_{\text {iso }}(\mathrm{H})$ (Table III). Using the $B_{K}$ method, a value of $-58.5 \mathrm{MHz}$ is calculated. It differs only $0.8 \mathrm{MHz}$ from the experimental value $(-57.7 \pm 0.3 \mathrm{MHz})$.

The success of the $B_{K}$ method can be understood in light of recent investigations performed for smaller systems, ${ }^{5,9}$ where we could show that the method is able to incorporate a large part of the indirect effect of the neglected configurations. These studies also show that most of the influence of higher than double excitation on spin properties is of indirect nature.

To test the MRCI/ $B_{K}$ method used in the present study, the isotropic hfecs were also calculated with various other methods, e.g., the unrestricted Hartree-Fock, (UHF) method, Møller-Plesset perturbation theory up to the fourth order (MP2-MP4), coupled cluster methods with and without an estimation of the triple excitations [CCD and $C C D(T)$ ], and the quadratic configuration interaction method ${ }^{27}$ with [QCISD $\left.(T)\right]$ and without an estimation of triple excitations (QCISD) as well. In the present study, all these treatments are based on UHF wave functions, while the RHF is the starting point for the MRCI/ $B_{K}$ method. All calculations were performed with the larger AO basis set (Table I) using the Gaussian88 program package. ${ }^{12} \mathrm{~A}$ comparison of the various theoretical methods is given in Table IV, which further includes results taken from the literature. For $A_{\text {iso }}\left({ }^{13} \mathrm{C}\right)$, the values calculated in the present study are also summarized in Fig. 3.

Let us first focus on the results for the hydrogen center. Except for UHF, which overestimates the absolute value of $A_{\text {iso }}(\mathrm{H})$ by about $26 \mathrm{MHz}$ and MP2 which shows 
TABLE IV. A comparison of isotropic hfec using various methods.

\begin{tabular}{|c|c|c|}
\hline Method & $\begin{array}{l}A_{100}\left({ }^{13} \mathrm{C}\right) \\
(\mathrm{MHz})\end{array}$ & $\begin{array}{l}A_{\text {ieo }}\left({ }^{\prime} \mathrm{H}\right) \\
(\mathbf{M H z})\end{array}$ \\
\hline \multicolumn{3}{|c|}{$\begin{array}{l}\text { Calculations performed in the present study } \\
\text { using the large basis set (see Table I) }\end{array}$} \\
\hline UHF & 100.4 & -84.4 \\
\hline MP2 & 37.4 & -62.1 \\
\hline MP3 & 31.4 & -58.0 \\
\hline MP4Dn & 29.5 & -56.7 \\
\hline MP4DQ & 32.1 & -57.2 \\
\hline MP4STQ & 35.0 & -57.6 \\
\hline $\mathrm{CCD}$ & 33.6 & -56.7 \\
\hline $\operatorname{CCD}(\mathrm{T})^{e}$ & 37.2 & -56.7 \\
\hline QCISD & 43.7 & -58.5 \\
\hline QCISD $(T)^{c}$ & 42.1 & -57.2 \\
\hline MRDCI & 37.2 & -56.3 \\
\hline $\mathrm{MRDCl}+B_{\mathrm{K}}$ & 45.8 & -58.5 \\
\hline \multicolumn{3}{|c|}{ Calculations taken from the literature } \\
\hline SDCL/STQ & 47.8 & -42.4 \\
\hline MBPT/STO & 37.3 & -59.4 \\
\hline MCSCF/num & 49.9 & -57.8 \\
\hline $\mathrm{CCD}(\mathrm{ST}) / \mathrm{CGTO}^{\mathrm{h}}$ & 36.6 & -57.0 \\
\hline SCL/CGTO & 41.4 & -57.1 \\
\hline SDCL/CGTO & 30.0 & -51.8 \\
\hline SDTCI/CGTO' & 45.8 & -58.0 \\
\hline \multicolumn{3}{|c|}{ Experimental data } \\
\hline & 46.8 & $-57.7^{k}$ \\
\hline & \pm 2.8 & \pm 0.3 \\
\hline
\end{tabular}

Only parts of the excitation classes are included.

'Full fourth order perturbation theory.

Large basis set using Slater functions (Ref. 28).

'Many-body perturbation theory (Ref. 29).

Numerical MCSCF, polarization effects are taken into account (Ref. 30).

Coupled cluster with estimates of triple and quadruple contributions $(1459 p 4 d 1 f, 9 s 3 p 1 d) \rightarrow[85 p 4 d 1 f, 6 s 3 p 1 d]$ (Ref. 11).

$'(10 s 6 p 2 d 1 f, 6 s 2 p 1 d) \rightarrow[6 s 3 p 2 d 1 f, 4 s 2 p 1 d]$ (Ref. 8).

'Reference 19.

${ }^{k}$ Reference 20.

a deviation of about $4 \mathrm{MHz}$, all methods give very similar results $(\sim-57.5 \mathrm{MHz})$. This behavior reflects the relative simplicity of the calculation as mentioned above.

The more difficult nature of $A_{\text {iso }}\left({ }^{13} \mathrm{C}\right)$ can be seen in Fig. 3. UHF overestimates $A_{\text {iso }}\left({ }^{13} \mathrm{C}\right)$ by more than a factor of 2 and only three methods predict values larger than 41 $\mathrm{MHz}$, i.e., a deviation of less than $10 \%$ from the experimental result. If MP2 is used, a value of $37.4 \mathrm{MHz}$ is found, but the value decreases to $31.6 \mathrm{MHz}$ if third order perturbation theory (MP3) is also included. By incorporating the fourth order (MP4STQ), the calculated value of $A_{\text {iso }}\left({ }^{13} \mathrm{C}\right)$ increases to $35.0 \mathrm{MHz}$, but it is still lower than the value obtained with MP2. All parts of the fourth order seem to be important, i.e., $A_{\mathrm{iso}}\left({ }^{13} \mathrm{C}\right)$ decreases to $29.5 \mathrm{MHz}$ if only double substitutions within the fourth order perturbation theory (MP4D) are accounted for. The dependence of $A_{\text {iso }}\left({ }^{13} \mathrm{C}\right)$ on the various orders of perturbation theory indicates that the good value obtained merely with MP2 is based on fortuitous error cancellation. The reasons for this behavior are still unknown. The $\mathrm{CC}$ methods used in the present study give values of $33.6 \mathrm{MHz}$ (CCD) and 37.2 $\mathrm{MHz}[\mathrm{CCD}(\mathrm{T})]$. These are in good agreement with the

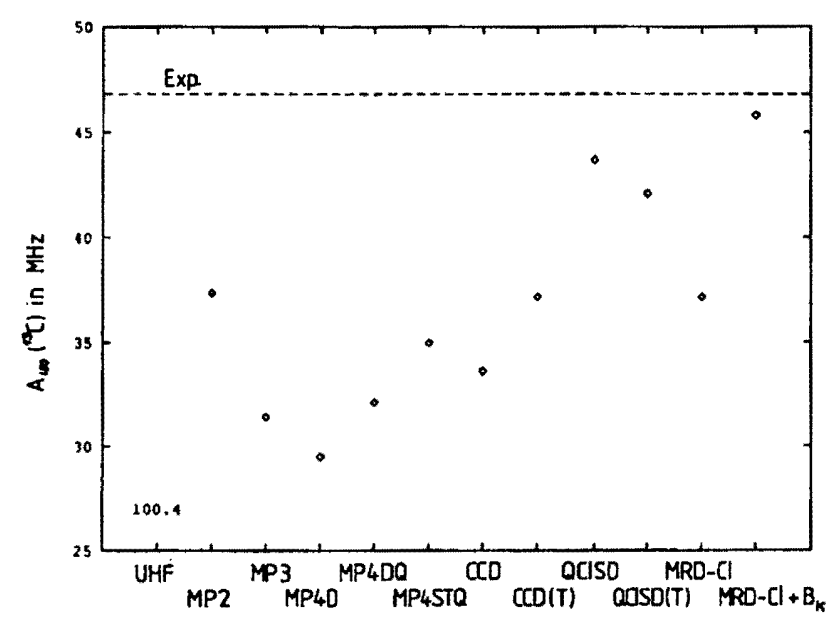

FIG. 3. $A_{\mathrm{ino}}\left({ }^{13} \mathrm{C}\right)$ of the $X^{2} \mathrm{II}$ of the $\mathrm{CH}$ molecule calculated with various methods.

$\mathrm{CCD}(\mathrm{ST})$ study by Carmichael (see the lower part of Table IV) who calculated $36.6 \mathrm{MHz}$ using the $\mathrm{CCD}(\mathrm{ST})$ and a basis set of similar quality. ${ }^{11}$ Besides the $\mathrm{MRCI} / \boldsymbol{B}_{\boldsymbol{K}}$ method presented here, only the QCISD method (43.7 $\mathrm{MHz}$ ) and the QCISD(T) method (42.1 MHz) are able to calculate $A_{\text {iso }}\left({ }^{13} \mathrm{C}\right)$ with an error of less than $10 \%$. It should be noted, however, that in the QCISD method, the inclusion of the triple excitations lowers $A_{\text {iso }}\left({ }^{13} \mathrm{C}\right)$ while opposite behavior is found for CCD calculation. The best value of the various methods presented in Fig. 3 is obtained by the $\mathrm{MRCI} / B_{K}$ method (45.8 $\mathrm{MHz}$ ).

As discussed above, the expense of the $B_{K}$ method should increase almost quadratically with the size of the $B_{K}$ space. The question of the dependence of the calculated value of a given property on the number of configurations actually corrected by the $B_{K}$ treatment is therefore quite important. In Fig. $4, A_{\text {iso }}\left({ }^{13} \mathrm{C}\right)$ is plotted as a function of

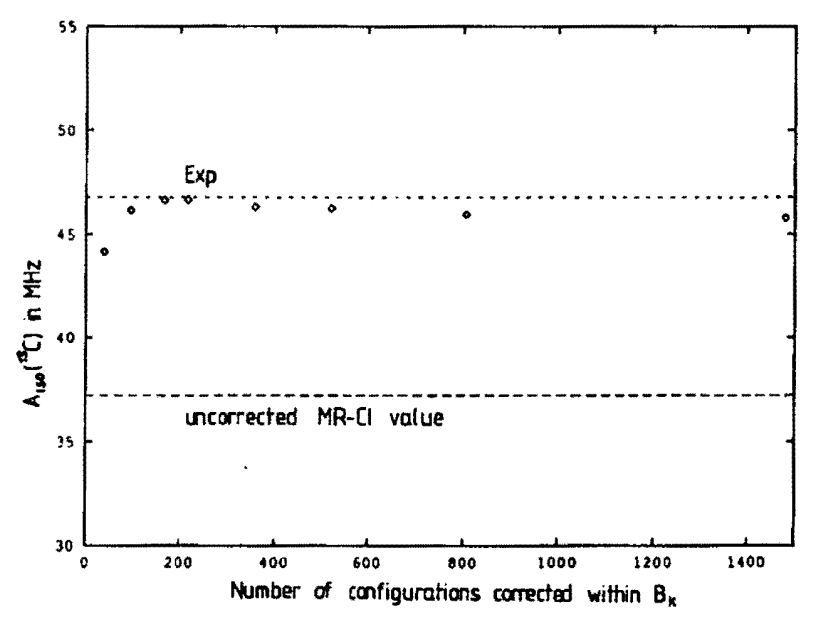

FIG. 4. $A_{\text {moo }}\left({ }^{13} \mathrm{C}\right)$ as a function of the size of the $B_{K}$ space. The foregoing MRCI calculation was performed with the large $A O$ basis set and 77 reference configurations. A total of 24135 configurations were handled variationally. The value obtained with the truncated MRCI wave function and the experimental value are given for comparison 
the size of the $B_{K}$ space. In the foregoing truncated MRCI calculation, 24135 configurations were handled variationally; the reference space consists of 77 configurations leading to a total MRCI space of 1154750 configurations. The isotropic hfecs obtained with the truncated MRCI wave function $\left(A_{\text {iso }}^{\text {CI }}\right)$ and the experimental values are given for comparison. The configurations included in the $B_{K}$ treatment were selected according to the magnitude of their coefficients in the truncated MRCI wave function. In Fig. 4 , it can be seen that for very small $B_{K}$ spaces $(<100$ configurations), the calculated value of $A_{\text {iso }}\left({ }^{13} \mathrm{C}\right)$ increases dramatically ( $\approx 9 \mathrm{MHz}$ ), while further extension of the $B_{K}$ space leads to much smaller variations $(<1 \mathrm{MHz})$ in $A_{\text {iso }}\left({ }^{13} \mathrm{C}\right)$. The fast convergence of $A_{\text {iso }}\left({ }^{13} \mathrm{C}\right)$ as a function of the number of configurations actually corrected in the $B_{K}$ treatment is obvious.

\section{SUMMARY}

In the present paper based on the $X^{2}$ II state of $\mathbf{C H}$, a detailed study of the MRCI $B_{K}$ method was undertaken. As expected, a reliable description of the isotropic hyperfine coupling constant ( $h f c c$ ) of the carbon center is a very difficult task for $a b$ initio calculations, while the spin density at the hydrogen center is much easier to obtain. In all calculations, the correction of the MRCI wave function by the $B_{K}$ method was found to reduce the error within the isotropic hfecs considerably. Using an appropriate $A O$ basis, the value obtained with $\mathrm{MRCI} / B_{K}$ is within the experimental uncertainties (MRCI/ $B_{K}-45.8 \mathrm{MHz}$; exp: -46.8 $\pm 2.8 \mathrm{MHz}$ ), while a value of $37.2 \mathrm{MHz}$ is found if no correction is performed. To test the $\mathrm{MRCL} / \boldsymbol{B}_{\boldsymbol{K}}$ method, the isotropic hfecs were also calculated with various other methods. Besides MRCI/ $B_{K}$, QCISD and QCISD(T) also give excellent results, while other methods such as MP2, MP3, MP4, and CCD(T) deviate more greatly. Finally, the spin densities were found to converge rapidly with respect to the number of configurations actually corrected within the $B_{K}$ treatment.

\section{ACKNOWLEDGMENTS}

The author would like to thank Professor S. D. Peyerimhoff and Professor M. Peric for many fruitful suggestions during the progress of this work and Dr. H.-U. Suter for his help with the MELDF-X calculations. The services and computer time of the Computing Center of the RWTH Aachen have been essential to the present study. The fi- nancial support granted to this work by the Deutsche Forschungsgemeinschaft in the framework of the project EN197/2-3 is gratefully acknowledged.

${ }^{1} \mathrm{R}$. McWeeny, Methods of Molecular Quantum Mechanics, 2nd ed. (Academic, New York, 1989).

${ }^{2}$ D. Feller and E. R. Davidson, J. Chem. Phys. 88, 7580 (1988).

${ }^{3}$ C. W. Bauschlicher, Jr, S. R. Langhoff, H. Partridge, and D. P. Chong, J. Chem. Phys. 89, 2985 (1988); C. W. Bauschlicher, Jr., ibid. 92,518 (1990).

${ }^{4}$ K. Funken, B. Engels, S. D. Peyerimhoff, and F. Grein, Chem. Phys. Lett. 172, 180 (1990).

${ }^{5}$ B. Engels, Chem. Phys. Lett. 179, 398 (1991).

${ }^{6}$ D. M. Chipman, I. Carmichael, and D. Feller, J. Phys. Chem. 95, 4702 (1991).

${ }^{7}$ I. Carmichael, J. Chem. Phys. 91,1072 (1989).

${ }^{8}$ D. M. Chipman, Theor. Chim. Acta 82, 93 (1992), and references cited therein.

${ }^{9}$ B. Engels, Theor. Chim. Acta 86, 429 (1993).

${ }^{10} \mathrm{Z}$. Gershgorn and I. Shavitt, Intn. J. Quantum Chem. 2, 751 (1968).

${ }^{11}$ I. Carmichael, J. Chem. Phys. 94, 5734 (1990).

${ }^{12}$ M. J. Frisch, M. Head-Gordon, H. B. Schlegel, K. Raghavachari, J. S. Binkley, C. Gonzalez, D. J. Defrees, D. J. Fox, R. A. Whiteside, R. Seeger, C. F. Melius, J. Baker, R. L. Martin, L. R. Kahn, J. J. P. Stewart, E. M. Fluder, S. Topiol, J. A. Pople, Gaussian, Inc., Pittsburgh, PA.

${ }^{13}$ P. O. Löwdin, J. Chem. Phys. 19, 1396 (1951).

${ }^{14} \mathrm{P}$. O. Löwdin, in Perturbation Theory and its Applications in Quantum Mechanics, edited by C. H. Wilcox (Wiley, New York, 1966).

${ }^{15}$ L. E. Nitzsche and E. R. Davidson, J. Chem. Phys. 68, 3103 (1977).

${ }^{16}$ E. R. Davidson, L. E. McMurchie, and S. I. Day, J. Chem. Phys. 74, 5491 (1981).

${ }^{17}$ D. C. Rawlings and E. R. Davidson, Chem. Phys. Lett. 98, 424 (1983).

${ }^{18}$ G. A. Segal and K. Wolf, Chem. Phys. 56, 321 (1981).

${ }^{19}$ T. C. Steimle, D. R. Woodward, and J. M. Brown, J. Chem. Phys. 85, 1276 (1986).

${ }^{20} \mathrm{C}$. R. Brazier and J. M. Brown, Can. J. Phys. 62, 1563 (1984).

${ }^{21}$ F. B. Duijneveldt, Technical Report RJ945, IBM Research Laboratories, San Jose, CA, 1971.

${ }^{22}$ MELDF-X was originally written by L. McMurchie, S. Elbert, S. Langhoff, and E. R. Davidson. It has been modified substantially by $D$. Feller, R. Cave, D. Rawlings, R. Frey, R. Daasch, L. Nitzche, P. Phillips, K. Iberle, C. Jackels, and E. R. Davidson.

${ }^{23}$ B. Engels and S. D. Peyerimhoff, Mol. Phys. 67, 583 (1989).

${ }^{24}$ R. J. Buenker and S. D. Peyerimhoff, Theor. Chim. Acta 35, 33 (1974); 39, 33 (1975); R. J. Buenker, S. D. Peyerimhoff, and W. Butscher, Mol. Phys. 35, 771 (1978).

${ }^{25}$ L. B. Knight, Jr., K. D. Johannessen, D. C. Cobranchi, E. A. Earl, D. Feller, and E. R. Davidson, J. Chem. Phys. 87, 885 (1987).

${ }^{26}$ B. Engels, S. D. Peyerimhoff, and E. R. Davidson, Mol. Phys. 62, 109 (1987).

${ }^{27}$ J. A. Pople, M. Head-Gordon, and K. Raghavachari, J. Chem. Phys. 87, 5968 (1987).

${ }^{28}$ C. F. Bender and E. R. Davidson, Phys. Rev. 183, 23 (1969).

${ }^{29} \mathrm{P}$. Kristiansen and L. Veseth, J. Chem. Phys. 84, 6336 (1986).

${ }^{30}$ D. M. Chipman, J. Chem. Phys. 91, 5455 (1989).

${ }^{31}$ D. M. Chipman, Theor. Chim. Acts 76, 73 (1989). 